\title{
Knockdown of Coronin-1C disrupts Rac1 activation and impairs tumorigenic potential in hepatocellular carcinoma cells
}

\author{
ZHI-GANG WANG ${ }^{1}$, MING-KU JIA ${ }^{1}$, HONG CAO $^{1}$, PENG BIAN $^{2}$ and XUE-DONG FANG ${ }^{1}$ \\ ${ }^{1}$ The Second Affiliated Hospital of Jilin University, Changchun, Jilin 130041; \\ ${ }^{2}$ Changchun Children's Hospital, Changchun, Jilin 130061, P.R. China
}

Received September 6, 2012; Accepted October 10, 2012

DOI: $10.3892 /$ or.2012.2216

\begin{abstract}
Coronin-1C is an important F-actin binding protein which is critical for cell motility. Furthermore, the expression of this protein was found to be increased in diffuse tumors and was correlated with the degree of tumor malignancy. However, the mechanism(s) through which this protein enhances malignancy in hepatocellular carcinoma (HCC) is poorly understood. In this study, we found that Coronin-1C was overexpressed in human HCC tissues compared with the adjacent non-tumor tissues. Overexpression of Coronin-1C enhanced the cell migration in the human HCC cell line BEL-7402, whereas suppressed cell migration and proliferation were observed in Coronin-1C-knockdown BEL-7402 cells together with impaired cell polarity, disrupted cytoskeleton and decreased Rac-1 activation. Moreover, the Coronin-1C knockdown cells displayed a lower degree of malignancy by inducing smaller tumors in nude mice. Thus, we demonstrated a relationship between Coronin-1C overexpression and human HCC growth through enhancement of tumor cell proliferation and migration, which are correlated with Rac-1 activation.
\end{abstract}

\section{Introduction}

Liver cancer is the third most common cause of death from cancer in the world. Hepatocellular carcinoma (HCC) is the most common type of liver cancer and accounts for $90 \%$ of primary liver cancer cases in China. The 5-year survival rate of liver cancer patients is low and is accompanied by a high recurrence rate after resection $(1,2)$. One major reason for disease recurrence originates from the metastasis of the primary tumor, tumor invasion and growth at a new site. The metastatic potential in recurrent tumors is determined in the original primary tumors $(3,4)$. Thus, the potential for metas-

Correspondence to: Dr Xue-Dong Fang, The Second Affiliated Hospital of Jilin University, 18 Ziqiang Road, Changchun, Jilin 130041, P.R. China

E-mail: xuedongfang11@gmail.com

Key words: Coronin-1C, hepatocellular carcinoma, proliferation, migration, Rac-1 tasis, invasion and growth could be used as parameters for judging the degree of tumor malignancy.

Migratory cancer cells display abnormal cell-cell and cellmatrix adhesion and an extensive remodeling of their actin cytoskeleton (5). The actin cytoskeleton is critical in triggering cell migration by actin network assembly and disruption (6). Tumor metastasis is attributed to a synergy of abnormal expression of genes in the primary tumor. The elements participating in actin network formation or regulating actin dynamics are associated with the metastatic potential of cancer cells. Coronin-1C, a protein with F-actin binding ability, was found to be overexpressed in cultured HCC cell lines with high metastatic potential when compared with cell lines with low potential (7).

Coronin was first identified in extracts of cells from Dictyostelium discoideum exhibiting an accumulation at the crown-like structures on the dorsal surface of these cells (8). Further studies on Coronin were carried out in eukaryotic organisms, and this protein as identified in mammalian animals was subdivided into three classes, type I, type II and type III. Although the expression of certain Coronin family members are tissue-specific, Coronin-1B and Coronin-1C, the two type I homologues, are ubiquitously expressed at high levels in most tissues with relatively lower levels for Coronin-1C (9) which localizes to the leading edge of cells (10). Most of the important insights into the functions of type I Coronin come from studies on Coronin-1A and Coronin-1B (11). Similar to other type I homologues, Coronin-1C binds to F-actin and interacts with the Arp2/3 complex (12). Moreover, the expression of Coronin-1C correlates with the degree of malignancy in human diffuse gliomas, and knockdown of Coronin-1C in glioblastoma cells was found to retard cell proliferation, motility and invasion (13). However, the functions of Coronin-1C in tumor cells have not been fully understood.

The Rho family of small GTPases are involved in cell motility by regulating cytoskeleton dynamics and cell adhesion (14,15). In HCC, Rho family small GTPases are implicated in tumorigenesis and metastasis (16). The migration and invasion of HCC are suppressed by reducing Racl expression (17). Yet, the relationship between Coronin-1C and Rac1 levels regarding tumor metastasis and growth remains unknown. In this study, we aimed to ascertain whether Coronin-1C expression levels in $\mathrm{HCC}$ are correlated with cell proliferation and motility by employing Coronin-1C-overexpressing and knockdown cell lines. We found that HCC cells with a lower 
Coronin-1C level displayed less motility, invasion and proliferation in vitro and less tumorigenetic potential in vivo in a nude mouse model. The decreased Coronin-1C expression was correlated with reduced Rac-1 activation.

\section{Materials and methods}

Clinical validation. Human HCC tissue and their corresponding adjacent non-tumorous specimens from 25 patients including 14 males and 11 females, mean age $60 \pm 5$ years, were obtained from The Second Affiliated Hospital of Jilin University, Changchun, China. All tumor tissues were subjected to analyses of the relative mRNA and protein levels of Coronin-1C, as well as to histological staining of Coronin-1C on sections.

Cell culture, plasmid construction, transient transfection and establishment of the stable knockdown cell line. Human HCC cell line BEL-7402 (18) was obtained from The Cell Bank of the Chinese Academy of Sciences, Shanghai, China. The cells were grown in RPMI-1640 medium containing 10\% fetal bovine serum (FBS) at $37^{\circ} \mathrm{C}$ in $5 \% \mathrm{CO}_{2}$. For the transient transfection, an expression plasmid containing full-length human Coronin-1C (aa 1-474; NM_014325) was generated as described (10). Then, $2 \times 10^{5}$ cells were seeded into a $35-\mathrm{mm}$ plate for $24 \mathrm{~h}$ to reach $\sim 75 \%$ confluence. Plasmids $(2 \mu \mathrm{g})$ were transfected using Lipofectamine 2000 transfection reagent (Invitrogen) according to the manufacturer's protocol. The cells with transiently overexpressed Coronin-1C were available for further experiments after an additional 48-h culture after transfection. For the establishment of the stable knockdown cell line, small hairpin RNAs (shRNAs) were used to specifically reduce the Coronin-1C expression in BEL-7402 cells. The shRNA oligo 5'-CGTCCACTACCTCAACACA TT-3' was cloned into pLKO.1-puro. Then the shRNA plasmids were co-transfected into 293TN cells with three lentiviral packaging plasmids, pVSV-G, pPACKH1-GAG and pPACKH1-REV. The resulting lentiviruses were collected and used to infect the target BEL-7402 cells. The cells were spread in 100-mm diameter plates $24 \mathrm{~h}$ after infection for a subsequent 7-day selection in RPMI-1640 medium containing $0.75 \mu \mathrm{g} / \mathrm{ml}$ puromycin. Cells infected with the pLKO.1-puro empty vector were regarded as the control transfectants. Transient Coronin-1C overexpression and stable knockdown of Coronin-1C expression were confirmed by western blot analysis with an anti-Coronin-1C antibody (Proteintech Group, Chicago, IL).

Cell viability assay. Cells $\left(5 \times 10^{4}\right.$ cells/well) were seeded in 24-well plates. The cell viability was determined at 12 and $48 \mathrm{~h}$ after seeding using an MTT assay. Briefly, cells were washed with PBS and replaced with $0.5 \mathrm{mg} / \mathrm{ml}$ MTT in serumfree medium for a further 3 -h incubation at $37^{\circ} \mathrm{C}$. Then the resulting formazan was extracted with isopropanol for a colorimetric measurement using a spectrophotometer (NanoDrop, Rockland, DE, USA) at $570 \mathrm{~nm}$ with the correction of interference at $690 \mathrm{~nm}$. For each group, four individual samples were collected and measured. The cell viability was indicated as arbitrary units of absorbance at $570 \mathrm{~nm}$.

Immunohistochemical staining assay. Tissues were fixed with 4\% paraformaldehyde, embedded in paraffin and sectioned.
The sections were stained with rabbit anti-Coronin-1C antibody (1:100, Proteintech Group) at $4^{\circ} \mathrm{C}$ overnight, and the secondary antibody for $2 \mathrm{~h}$ at room temperature. The sections were finally visualized using a DAB substrate chromogen system (DakoCytomation, Denmark) according to the manufacturer's instructions.

Western blot analysis. Cells were washed in PBS and lysed in RIPA buffer (20 mM Tris, pH 7.4, $150 \mathrm{mM} \mathrm{NaCl,} 1 \%$ Triton $\mathrm{X}-100,1 \%$ Na deoxycholate, 2 mM EGTA, 2 mM EDTA, $0.1 \%$ SDS) containing protease inhibitor cocktail (1:100; Sigma) and phosphatase inhibitor cocktails 1 and 2 (both at 1:200; Sigma). Lysates were centrifuged at $14,000 \times \mathrm{g}$ for $10 \mathrm{~min}$ at $4^{\circ} \mathrm{C}$ and the total protein concentrations were equalized with BCA protein quantification assay. Then equal total protein $(30 \mu \mathrm{g})$ for each sample was loaded onto SDS-PAGE gel and blotted with different primary antibodies (1:500, rabbit antiRac-1 antibody; Santa Cruz Biotechnology, Santa Cruz, CA; 1:1000, mouse anti-GAPDH; Santa Cruz Biotechnology; 1:500, rabbit anti Coronin-1C, Proteintech Group) at $4^{\circ} \mathrm{C}$ overnight and the corresponding secondary antibodies. Finally, the results were visualized with enhanced chemiluminescence (ECL).

RNA extraction and semi-quantitative RT-PCR. Total RNA was extracted from the cells or tissues with the RNeasy Mini kit (Qiagen, Valencia, CA) following the manufacturer's instructions. Reverse transcription into cDNA was carried out using $500 \mathrm{ng}$ of total RNA using the SuperScript III First-Strand Synthesis System for RT-PCR (Invitrogen). PCR was carried out with the following primer pairs and Power SYBR-Green Master kit according to the manufacturer's protocol: GAPDH (forward, 5'-AAG GTG AAG GTC GGA GTC AAC-3'; reverse, 5'-GGG GTC ATT GAT GGC AAC AAT A-3'); Coronin-1C (forward, 5'-GCA GAA GAG TGG TTC GAA GG-3'; reverse, 5'-TGA TCA GGT CGC ACT TCT TG-3').

Wound healing assay. BEL-7402 cells $\left(2 \times 10^{5}\right)$ were seeded into 35-mm dishes and grown in RPMI-1640 medium supplemented with $1 \%$ FBS overnight to form a confluent monolayer. A wound was produced on the following day by scraping the cells with a pipette tip. The cells were allowed to recover for 15 min and replenished with $10 \%$ FBS to drive cell migration.

Transwell migration assay. Cell invasion assay was performed with self-coated Matrigel (BD Biosciences, Sparks, MD) on the upper surface of a Transwell chamber. The cells that had invaded through the extracellular matrix layer to the lower surface of the membrane were fixed with methanol and stained with DAPI. Images of three randomly selected fields of the fixed cells were captured, and the cells were counted. Experiments were repeated independently three times.

Co-staining of Golgi apparatus and $\alpha$-actin. The Golgi apparatus reorientation assay was performed as previously described (19). Briefly, wounds were created on a monolayer of BEL-7402 cells seeded on coverslips. Cells were then allowed to migrate for $5 \mathrm{~h}$ and stained with an anti- $\alpha$-actin antibody 
(Clone 5C5; Santa Cruz Biotechnology) and a corresponding secondary antibody, $10 \mathrm{~g} / \mathrm{ml}$ lectin HPA Alexa Fluor 488 conjugates (Invitrogen) and DAPI to indicate the positions of actin, Golgi apparatus and the nucleus, respectively. The migrating cells at the wound edge were determined to be positive when the Golgi apparatus was located in front of the nucleus in the direction towards the wound. At least 300 cells were counted in randomly chosen fields under a fluorescence microscope. The experiments were repeated independently three times.

Actin staining with FITC-conjugated phalloidin. Cells were seeded on $22 \times 22 \mathrm{~mm}$ coverslips and then fixed in $3 \%$ paraformaldehyde. The cells were then stained with FITC-conjugated phalloidin (Sigma) according to the manufacturer's instructions. Finally, images were acquired with a Zeiss LSM 510 Meta confocal microscope.

Racl GST-PAK pull-down assay. A glutathione-S-transferase (GST)-PAK-CD (PAK-CRIB domain) fusion protein, containing the Rac1 binding region from human PAK1B, was used to determine Rac1 activity as previously described (20). Escherichia coli transformed with the GST-PAK-CD construct were grown at $37^{\circ} \mathrm{C}$ to an absorbance of 0.3. Expression of recombinant protein was induced by culturing with $0.1 \mathrm{mmol} / 1$ isopropylthiogalactoside for $2 \mathrm{~h}$. Cells were homogenized, resuspended in lysis buffer [Tris- $\mathrm{HCl}$ $50 \mathrm{mmol} / \mathrm{l}$ (pH 7.4), $\mathrm{NaCl} 100 \mathrm{mmol} / \mathrm{l}, \mathrm{MgCl}_{2} 2 \mathrm{mmol} / \mathrm{l}$, benzamidine $1 \mathrm{mmol} / \mathrm{l}, \mathrm{NP}-401 \%$, glycerol $10 \%$, leupeptin, pepstatin and aprotinin $1 \mu \mathrm{g} / \mathrm{ml}$, respectively] and centrifuged at $21,000 \mathrm{rpm}$ for $5 \mathrm{~min} 4^{\circ} \mathrm{C}$. Equal amounts of supernatant protein were incubated with the GST-PAK-CD fusion protein bound to glutathione-coupled Sepharose beads at $4^{\circ} \mathrm{C}$ for $30 \mathrm{~min}$. Beads were washed three times with lysis buffer, eluted in loading buffer $[60 \mathrm{mmol} / \mathrm{l}$ Tris ( $\mathrm{pH} 6.8), 2 \%$ sodium dodecylsulfate, $10 \%$ glycerol, $0.1 \%$ bromphenol blue], and analyzed for bound Rac1 by western blotting.

Tumor assay in nude mice. The nude mice were purchased from Shanghai SLAC Laboratory Animal Co., Ltd., and maintained under SPF environmental conditions. All experimental protocols were approved by the Animal Care and Use Committee of Jilin University. In tumor xenograft experiments, shCoronin-1C cells and control transfectants were subcutaneously injected into nude mice. The tumor size was measured once every week until the mice were sacrificed 5 weeks after injection. Then each primary tumor was weighed.

Statistical analysis. Data from at least three independent experiments were analyzed with the two-tailed Student's t-test and are represented as the means \pm SD. ' $n$ ' means the sample size, namely the number of the nude mice that received the subcutaneous cell injection. P-values $\leq 0.05$ were considered to indicate a statistically significant difference.

\section{Results}

Coronin-1C is increased in hepatocellular carcinoma tissues. The Coronin-1C mRNA levels (via semi-quantitative RT-PCR)
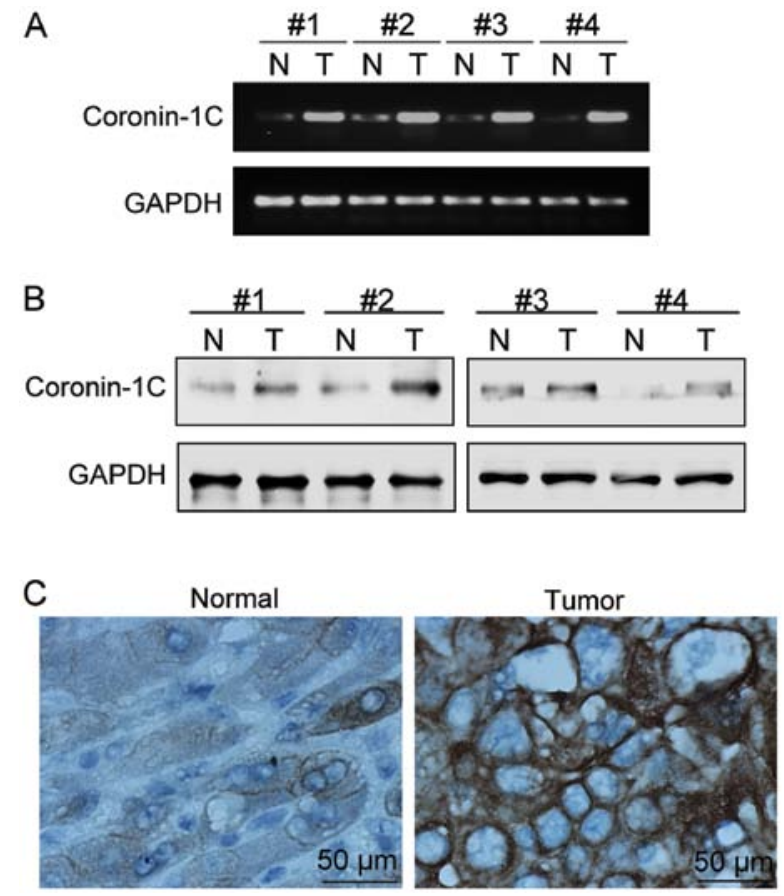

Figure 1. Coronin-1C overexpression in HCC patient tissue specimens. The HCC tumor and the corresponding non-tumorous adjacent tissues were obtained from hepatectomy. Coronin-1C mRNA and protein levels in these tissues were determined by semi-quantitative PCR and western blot analysis, respectively. (A) mRNA levels of Coronin-1C from four representative tissue pairs. (B) Expression levels of Coronin-1C protein from four representative tissue pairs. T, human HCC tissues. $\mathrm{N}$, corresponding non-tumorous adjacent tissues. (C) Coronin-1C overexpression in HCC tissues was confirmed by immunohistological staining with an anti-Coronin-1C antibody.

and protein levels (via both western blot analysis and immunohistological staining of Coronin-1C) were detected in paired specimens of human HCC tissues and their corresponding non-tumorous adjacent tissues from 25 patients (14 male and 11 female, mean age $60 \pm 5$ years). Coronin- $1 \mathrm{C}$ was found to be overexpressed at the mRNA and protein levels in $22(13$ males and 9 females) and 20 (12 males and 8 females) HCC cases, respectively. The representative results from 4 patients are shown in Fig. 1A and B. In the immunohistological assay with the anti-Coronin-1C antibody, highly positive Coronin-1C staining was noted in the tumor tissues but not in the adjacent non-tumorous tissues (Fig. 1C). Furthermore, patient gender was not associated with the tendency of increased Coronin-1C in HCC.

Coronin-1C level is related with the degree of malignancy of BEL-7402 cells. BEL-7402 is a cell line derived from human HCC (18). In order to observe the effects of Coronin-1C on BEL-7402 cell behavior related to tumor malignancy, including cell migration, invasion and proliferation, we transiently transfected BEL-7402 cells with transfecting plasmids containing full-length human Coronin-1C causing overexpression in the BEL-7402 cells. The cells transfected with the vector plasmids were taken as control transfectants for transient overexpression (CTT). A BEL-7402 cell line with stable knockdown of Coronin-1C (shCoronin-1C), as well as its corresponding control transfectant (CT), was also employed. All transfectants were submitted to western blot analysis to detect the 
A

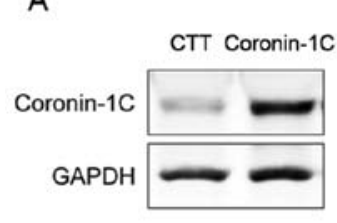

B

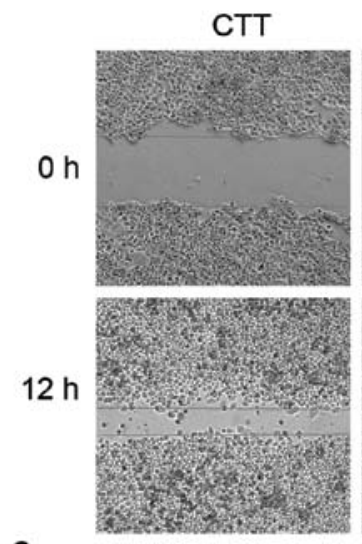

C

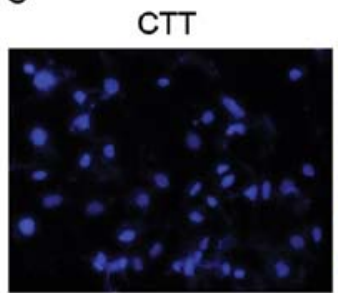

CT

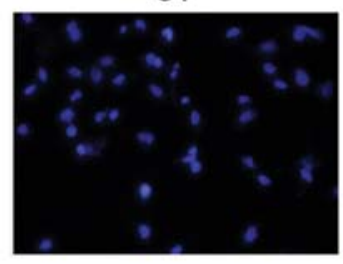

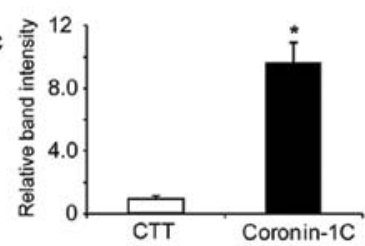

Coronin-1C
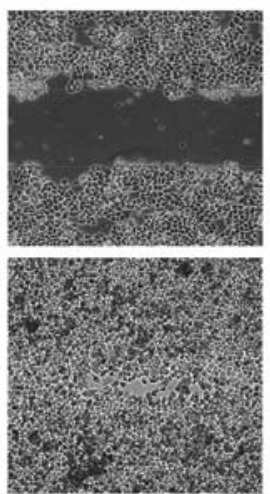

Coronin-1C

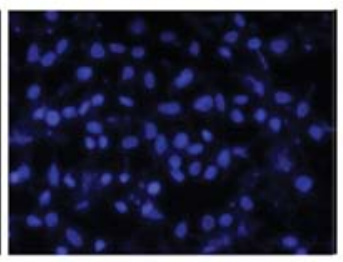

shCoronin-1C

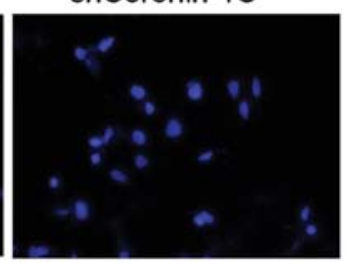

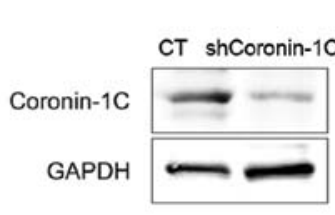

CT

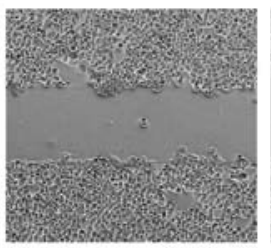

shCoronin-1C
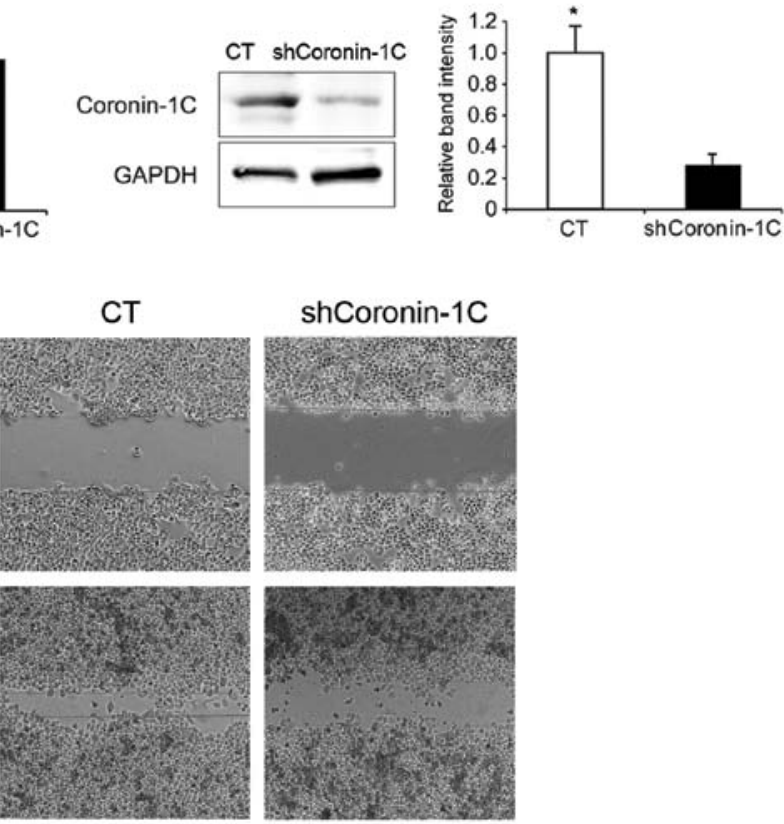

D
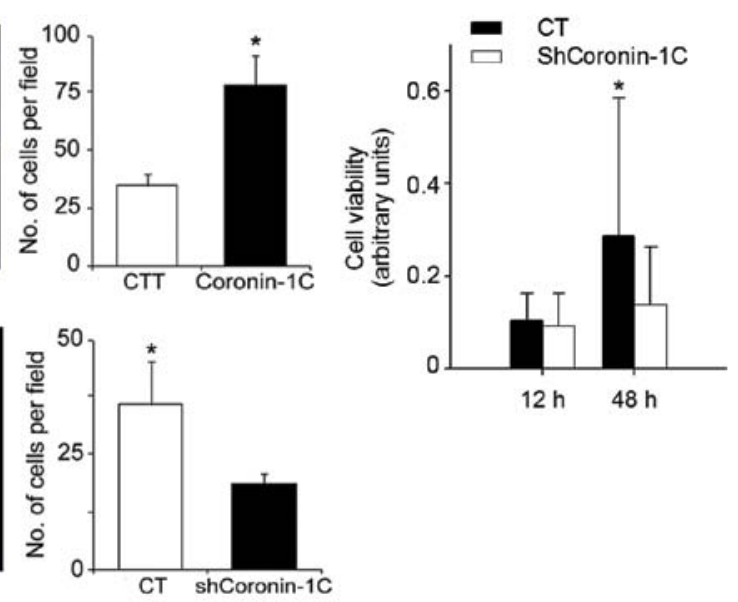

Figure 2. Coronin-1C overexpression enhances HCC cell migration, invasion and proliferation. (A) The transient overexpression of full-length Coronin-1C or stable knockdown of Coronin-1C in BEL-7402 cells was determined by western blot analysis. The Coronin-1C expression levels were normalized to the levels of the corresponding internal control GAPDH by band intensity determination from three independent experiments. (B) A monolayer of the control transfectants (CTT and CT), cells with transient full-length Coronin-1C overexpression (Coronin-1C) and cells with stable knockdown of Coronin-1C (shCoronin-1C) were subjected to scratch wounding. The images were captured at 0 and $12 \mathrm{~h}$ after wounding. (C) Coronin-1C-overexpressing cells $\left(3 \times 10^{5}\right)($ Coronin-1C), shCoronin-1C cells or their corresponding control tranfectants (CTT and CT) were seeded onto the upper surface of a Matrigel-coated membrane in a Transwell chamber and allowed to migrate for $24 \mathrm{~h}$. The cells that invaded through the extracellular matrix layer to the lower surface of the membrane were fixed with methanol and stained with DAPI. Images of three randomly selected fields of the fixed cells were captured, and total cells in each field were counted.

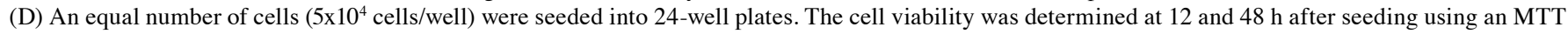
assay. The cell viability was expressed as arbitrary units of absorbance at $570 \mathrm{~nm}$ (CTT, control transfectant for transient Coronin $1 \mathrm{C}$ overexpression; CT, control transfectant for shCoronin-1C) ( $\mathrm{P}<0.05)$.

Coronin-1C levels. Compared with the corresponding control transfectant, Coronin-1C-overexpressing BEL-7402 cells and shCoronin-1C cells displayed a 9-fold increase and a $60 \%$ decrease in Coronin-1C levels, respectively (Fig. 2A). For the wound healing assay, the cells were seeded and after $24 \mathrm{~h}$ were subjected to a scratch-wound. At $18 \mathrm{~h}$ after wounding, the cells were observed and evaluated for their cellular ability to close the scratched area. In the Coronin-1C-overexpressing cells, the scratched area was occupied by migrating cells to a $60-80 \%$ confluence. The wound of the control CTT cells remained unmerged but showed a reduced area between the two wound edges. However, the wound area in the shCoronin-1C cells was larger than the area in the corresponding control cells (CT). This indicates a slower cell migration in the shCoronin-1C cells (Fig. 2B).

Similar to the results of the scratch healing assay, the cells overexpressing Coronin-1C displayed rapid invasion through the extracellular matrix layer to the lower surface of the membrane in the Transwell migration assay. The slowest invasion was observed in the shCoronin-1C cells (Fig. 2C). However, in the cell viability assay, lower Coronin-1C expression correlated with a lower cell proliferation rate in the shCoronin-1C cells (Fig. 2D). These data clearly indicate that Coronin-1C enhances cell motility and cell proliferation in HCC cells. 
A
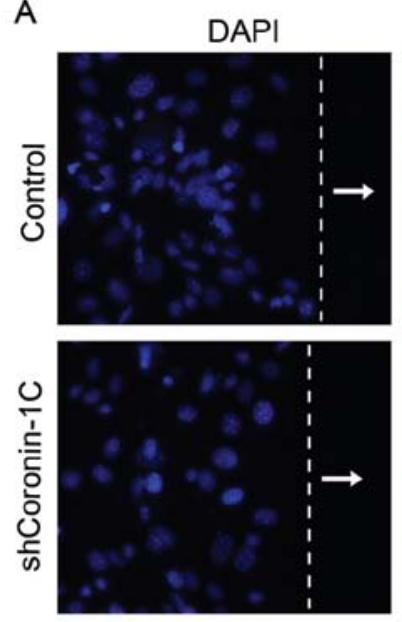

B

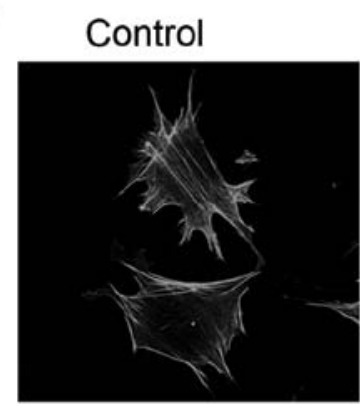

Golgi apparatus
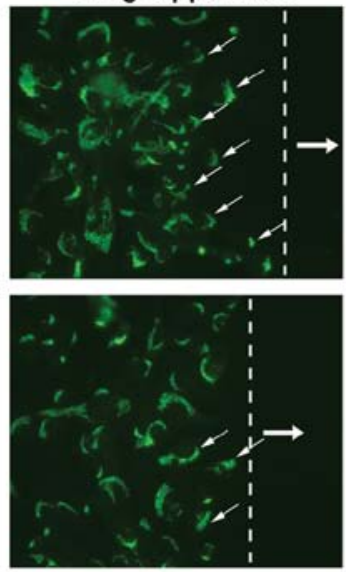

shCoronin-1C

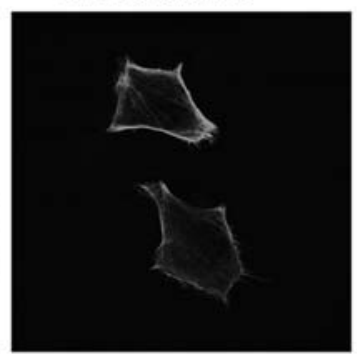

F-actin
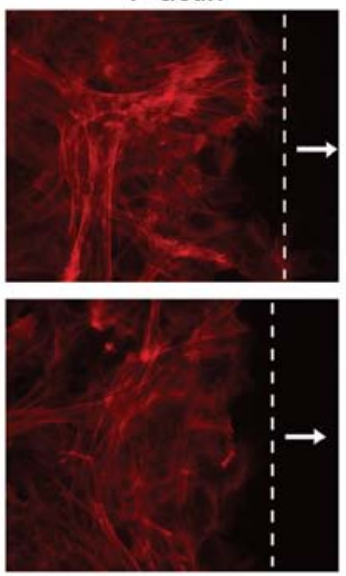

Merged
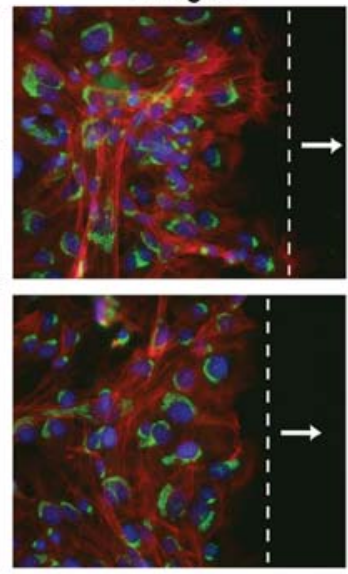

C

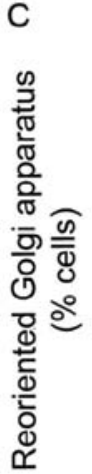

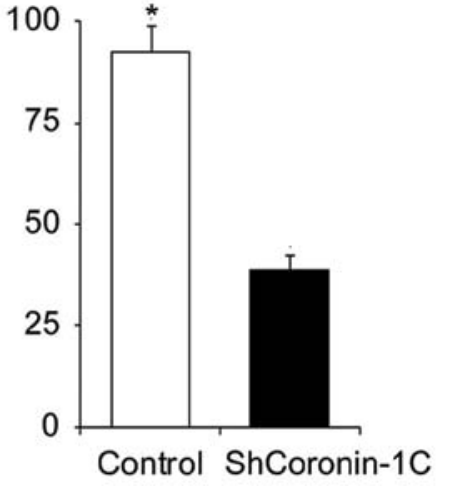

Figure 3. Reduced Golgi apparatus realignment and disrupted cell skeleton in shCoronin-1C cells. Cells were seeded on coverslips and one scratch wound was made in the monolayer of cells. The cells were then fixed and subjected to staining of the Golgi apparatus (lectin HPA Alexa Fluor 488 conjugates), $\alpha$-actin (an anti- $\alpha$-actin antibody) and nucleus (DAPI). At the wound edge, the cells exhibiting a Golgi apparatus located to the front of the nucleus in the direction of the wound were considered as Golgi apparatus realignment-positive cells. (A) The Golgi apparatus positions and the actin filaments were stained, respectively. The wounds are indicated with white lines, and thick arrows indicate the direction of cell migration. The thin arrows indicate the Golgi apparatus realignmentpositive cells. (B) The stress fiber network (polymerized actin) was stained with FITC-conjugated phalloidin. (C) The percentage of cells with realigned Golgi apparatus in total cells ( ${ }^{*} \mathrm{P}<0.05$; control transfectant vs. shCoronin-1C transfectant).

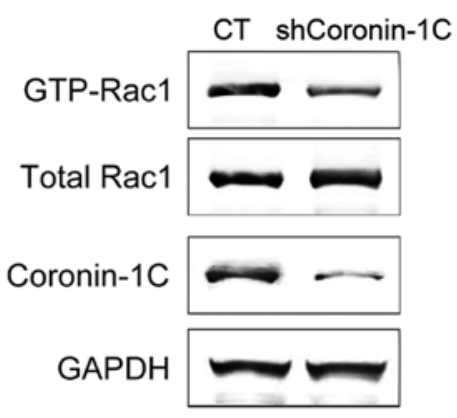

Figure 4. Attenuated Rac-1 activation in shCoronin-1C cells. Equal amount of total cell lysates from control transfectant (control) or shCoronin-1C cells were subjected to a GST-pull down assay using recombinant protein (GST)PAK-CD. The total Rac-1 levels (total Rac-1), total GAPDH levels (GAPDH) and the total Coronin-1C levels (Coronin-1C) in lysates, as well as the bound Rac-1 levels (GTP-Rac1) were analyzed by western blot analysis.

Knockdown of Coronin-1C reduces cell polarity and disrupts the cytoskeleton. To ascertain whether Coronin-1C affects cell polarity, we wounded the adherent shCoronin-1C and control monolayer cells and observed the cellular morphology and the positions of the Golgi apparatus in the cells at the scratch edge. Compared with the control cells, the shCoronin-1C cells displayed a reduced stress fiber network (Fig. 3A), consistent with changes in the cell morphology showing less lamellipodial extensions (Fig. 3B). The percentage of cells exhibiting Golgi apparatus realignment, located in front of the nucleus towards the direction of the scratch edge, was determined. Decreased Golgi apparatus realignment was found in the shCoronin-1C cells but not in the control transfected cells (Fig. 3C). These observations suggest that Coronin-1C participates in cytoskeleton formation or stability which contributes to enhancement of cell motility.

Attenuated Rac-1 activation in Coronin-1C-knockdown cells. Coronin-1A was reported to promote Rac-1 translocation and activation (21). Rac-1 activation was associated with the migration and tumor metastasis in HCC (22). In shCoronin-1C cells, the level of activated Rac-1, by means of GTP bound Rac-1, was reduced. Yet, the total Rac-1 protein level was not affected by diminished Coronin-1C (Fig. 4). Thus, Coronin-1C overexpression in HCC cells enhanced Rac-1 activation by triggering the interaction between GTP and Rac-1 but not by altering the Rac-1 protein expression. 


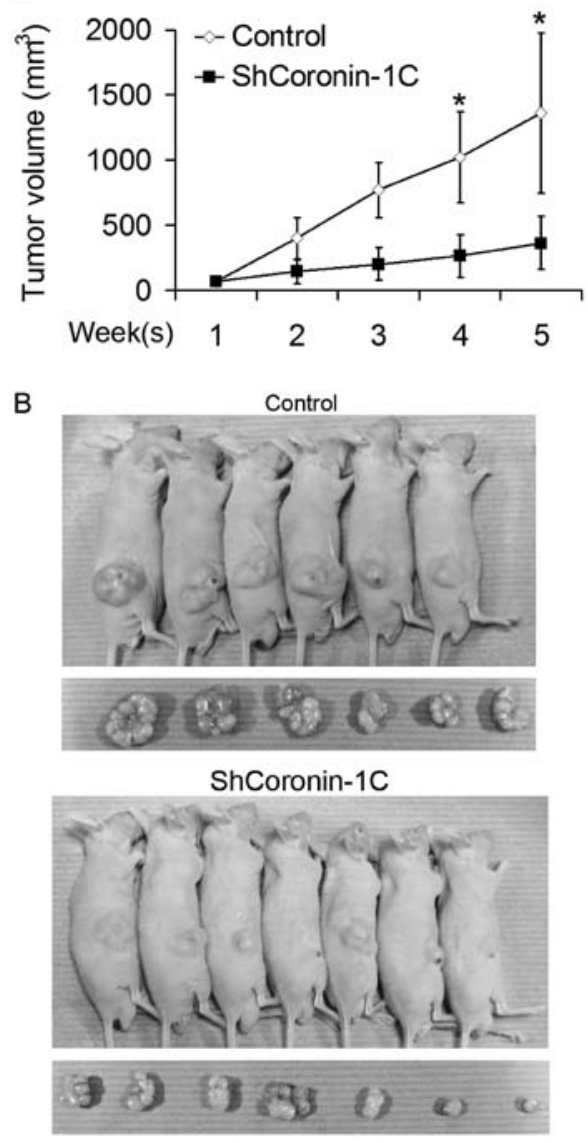

C

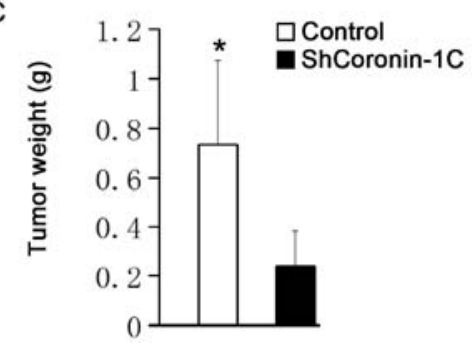

Figure 5. shCoronin-1C cells display less tumorigenetic potential shCoronin-1C and control transfectants were subcutaneously injected into nude mice ( $\mathrm{n}=7$ for shCoronin-1C group and $\mathrm{n}=6$ for control group). The tumor size was measured once every week until the mice were sacrificed 5 weeks after the injection. The tumors were then weighed and photographed. (A) The xenograft tumor growth curve for 5 weeks ( $\mathrm{P}<0.5$ for shCoronin- $1 \mathrm{C}$ mice vs. control mice at each time point). (B) After sacrifice, the tumor-bearing nude mice and the xenograft tumors were photographed to indicate the solid tumor growth. (C) Each primary tumor in the two groups was weighed, and the difference between the two groups was evaluated with the Student's t-test $\left({ }^{*} \mathrm{P}<0.5\right.$, shCoronin- $1 \mathrm{C}$ mice vs. control mice).

Tumorigenic potential in Coronin-1C-knockdown BEL-7402 cells. The attenuated malignant potential of BEL-7402 cells by Coronin-1C knockdown was also confirmed in vivo. shCoronin-1C and control transfected cells were subcutaneously injected into nude mice. The xenograft tumor volumes in nude mice were measured once a week, and the mice were sacrificed for tumor weighing 5 weeks afterwards. The xenograft tumor growth curve is shown in Fig. 5A. Larger tumor volume were observed in mice injected with the control-transfected cells even one week after the injection. Differences in tumor extension between the shCoronin-1C and control mice, including tumor volume and weight measured after sacrifice, were noted five weeks after the injection (Fig. 5B and C). These results indicate that Coronin-1C silencing reduces the rapid tumor growth in vivo.

\section{Discussion}

Hepatocellular carcinoma (HCC) is the third most common cause of cancer-related mortality in the world (23). Most of the HCC cases occur in Asia and sub-Saharan Africa (24). The hospital mortality rate of hepatectomy for HCC has improved in past 20 years; however, the long-term survival rate remains unsatisfactory (2). Metastasis is a major cause of high mortality in HCC patients after surgical resection. More and more biomarkers to determine the degree of tumor malignancy and metastasis have been identified to cope with this challenge (25). Coronin-1C was considered to be a biomarker for HCC cells with high metastatic potential. We found Coronin-1C protein levels were increased in 20 of the $25 \mathrm{HCC}$ patient specimens suggesting that an increase in Coronin-1C tumors may be a general biomarker for $\mathrm{HCC}$ occurrence. Although the functions for Coronin-1C are not fully understood, studies of other type I Coronin homologues have indicated that this protein is able to bind F-actin and acts as an inhibitor for the Arp2/3 complex $(10,12)$. Overexpression of Coronin-1B mutants causing enhanced Arp2/3 interaction led to increased cell motility, whereas overexpression of mutants containing less interactional affinity suppressed cell motility. Coronin-Arp2/3 interaction was found to be regulated by Coronin phosphorylation (26). By cooperating with Arp2/3 activator WASP or its more widely expressed homologue N-WASP, type I Coronins regulate Arp2/3 nucleation tightly and precisely (27-29). These results have demonstrated that the regulative functions of type I Colonin proteins on cell motility depend on modulating the Arp2/3 complex.

During cell migration, a cell first extends its protrusions such as lamellipodia and filopodia to the front, forms adhesion at the leading edge and finally retracts its trail at the trailing edge. This process results from the treadmilling of actin filaments based on F-actin dynamics: F-actin assembly followed by F-actin extension and degradation (6). The proteins regulating the actin filament polymerization and depolymerization control the force on protrusions. One hypothesis suggests that Coronin is a coordinating factor between Arp2/3-based actin assembly and Cofilin-mediated disassembly $(28,30)$. Accumulation of Coronin 1A was associated with more rapid F-actin dynamics (31). As an inhibitor to Arp2/3 activation, Coronin replaces Arp2/3 at the actin branch, facilitates actin debranching or creates more flexible actin branches which consequentially guarantees the recycling of actin monomers in the middle and rear of the lamellipodium and supports the actin assembly at the front (32). More flexible actin branches may contribute to the genesis of stress fiber network. These previous studies offer relevant explanations for the reduced stress fiber network noted in Coronin-1C-depleted cells.

Rac-1 is a Rho family small GTPase participating in many cell events including cell growth, cytoskeletal reorganization, cell migration and invasion (33). A negative microRNA for RAC1 was found to diminish Rac-1 expression and suppress migration and invasion of HCC cells (17). This may explain 
why the proliferation rate was reduced in shCoronin-1C cells which have less activated Rac-1. The activation of Rac-1 depends on its translocation from the cytosol to the membrane and release from its inhibitor RhoGDI (34). The manner by which Coronin-1C modulates Rac-1 activation might be similar to its homologue Coronin-1A, which facilitates Rac-1 activation by promoting Rac-1 membrane translocation and dissociation from RhoGDI $\alpha$ via a cytoskeleton-based feedback loop (21). What is more, another possible effect of Coronin-1C on modulating cell motility is its affect on Arp2/3 activator WASP or N-WASP. It is not yet clear whether there are direct interactions between WASP/N-WASP and Rho family small GTPases or not. Yet, WASP and N-WASP were reported to be activated by Rho family small GTPase Cdc 42 and Rac-1. Rac-1 displayed a more potent affect for activating N-WASP (35).

In this study, we found that Coronin-1C was overexpressed in hepatocellular carcinoma tissues. HCC cells displayed enhanced cell migration, invasion and proliferation which were impaired in Coronin-1C-deficient cells with a coordinated attenuation in Rac-1 activation. The upregulated Coronin-1C in tumor cells enhanced the metastasis by promoting a more rapid actin dynamics which was mediated by Arp2/3 complex inhibition and the events relating to Rac-1 activation. Moreover, overexpression of Coronin-1C triggered a more rapid proliferation rate in HCC cells. All these changes endow HCC cells with high tumorigenic potential. Thereby, Coronin-1C may be used as a diagnostic marker for tumor malignancy and a target for tumor therapy in the future.

\section{Acknowledgements}

This study was supported by a grant from the Jilin Provincial Science and Technology Department, no. 200705199.

\section{References}

1. Jemal A, Thomas A, Murray T and Thun M: Cancer statistics, 2002. CA Cancer J Clin 52: 23-47, 2002.

2. Fan ST, Mau Lo C, Poon RT, et al: Continuous improvement of survival outcomes of resection of hepatocellular carcinoma: a 20-year experience. Ann Surg 253: 745-758, 2011.

3. Tang ZY, Ye SL, Liu YK, et al: A decade's studies on metastasis of hepatocellular carcinoma. J Cancer Res Clin Oncol 130 187-196, 2004.

4. Tang Z, Zhou X, Lin Z, et al: Surgical treatment of hepatocellular carcinoma and related basic research with special reference to recurrence and metastasis. Chin Med J 112: 887-891, 1999.

5. Yilmaz M and Christofori G: Mechanisms of motility in metastasizing cells. Mol Cancer Res 8: 629-642, 2010.

6. Bugyi B and Carlier MF: Control of actin filament treadmilling in cell motility. Annu Rev Biophys 39: 449-470, 2010.

7. Wu L, Peng CW, Hou JX, et al: Coronin-1C is a novel biomarker for hepatocellular carcinoma invasive progression identified by proteomics analysis and clinical validation. J Exp Clin Cancer Res 29: 17, 2010

8. de Hostos EL, Bradtke B, Lottspeich F, Guggenheim R and Gerisch G: Coronin, an actin binding protein of Dictyostelium discoideum localized to cell surface projections, has sequence similarities to G protein beta subunits. EMBO J 10: 4097-4104, 1991.

9. Uetrecht $\mathrm{AC}$ and Bear JE: Coronins: the return of the crown. Trends Cell Biol 16: 421-426, 2006.

10. Spoerl Z, Stumpf M, Noegel AA and Hasse A: Oligomerization, F-actin interaction, and membrane association of the ubiquitous mammalian coronin 3 are mediated by its carboxyl terminus. J Biol Chem 277: 48858-48867, 2002.
11. Chan KT, Creed SJ and Bear JE: Unraveling the enigma: progress towards understanding the coronin family of actin regulators. Trends Cell Biol 21: 481-488, 2011.

12. Rosentreter A, Hofmann A, Xavier CP, Stumpf M, Noegel AA and Clemen CS: Coronin 3 involvement in F-actin-dependent processes at the cell cortex. Exp Cell Res 313: 878-895, 2007.

13. Thal D, Xavier CP, Rosentreter A, et al: Expression of coronin-3 (coronin-1C) in diffuse gliomas is related to malignancy. J Pathol 214: 415-424, 2008.

14. Parsons JT, Horwitz AR and Schwartz MA: Cell adhesion: integrating cytoskeletal dynamics and cellular tension. Nat Rev Mol Cell Biol 11: 633-643, 2010.

15. Spiering D and Hodgson L: Dynamics of the Rho-family small GTPases in actin regulation and motility. Cell Adh Migr 5: 170-180, 2011.

16. Xue Y, Bi F, Zhang X, et al: Role of Rac1 and Cdc42 in hypoxia induced p53 and von Hippel-Lindau suppression and HIF1alpha activation. Int J Cancer 118: 2965-2972, 2006.

17. Wu L, Cai C, Wang X, Liu M, Li X and Tang H: MicroRNA$142-3$ p, a new regulator of RAC1, suppresses the migration and invasion of hepatocellular carcinoma cells. FEBS Lett 585: 1322-1330, 2011.

18. Chen R, Zhu D, Ye X, Shen D and Lu R: Establishment of three human liver carcinoma cell lines and some of their biological characteristics in vitro. Sci Sin 23: 236-247, 1980.

19. Wong CC, Wong CM, Tung EK, Man K and Ng IO: Rho-kinase 2 is frequently overexpressed in hepatocellular carcinoma and involved in tumor invasion. Hepatology 49: 1583-1594, 2009.

20. Maack C, Kartes T, Kilter H, et al: Oxygen free radical release in human failing myocardium is associated with increased activity of rac1-GTPase and represents a target for statin treatment. Circulation 108: 1567-1574, 2003.

21. Castro-Castro A, Ojeda V, Barreira M, et al: Coronin 1A promotes a cytoskeletal-based feedback loop that facilitates Rac1 translocation and activation. EMBO J 30: 3913-3927, 2011.

22. Chang CY, Lin SC, Su WH, Ho CM and Jou YS: Somatic LMCD1 mutations promoted cell migration and tumor metastasis in hepatocellular carcinoma. Oncogene 31: 2640-2652, 2011.

23. Parkin DM, Bray F, Ferlay J and Pisani P: Global cancer statistics, 2002. CA Cancer J Clin 55: 74-108, 2005.

24. Sell S: Mouse models to study the interaction of risk factors for human liver cancer. Cancer Res 63: 7553-7562, 2003.

25. Gonzalez SA and Keeffe EB: Diagnosis of hepatocellular carcinoma: role of tumor markers and liver biopsy. Clin Liver Dis 15: 297-306, vii-x, 2011.

26. Cai L, Holoweckyj N, Schaller MD and Bear JE: Phosphorylation of coronin $1 \mathrm{~B}$ by protein kinase $\mathrm{C}$ regulates interaction with Arp2/3 and cell motility. J Biol Chem 280: 31913-31923, 2005.

27. Machesky LM, Reeves E, Wientjes F, et al: Mammalian actinrelated protein $2 / 3$ complex localizes to regions of lamellipodial protrusion and is composed of evolutionarily conserved proteins. Biochem J 328: 105-112, 1997.

28. Cai L, Marshall TW, Uetrecht AC, Schafer DA and Bear JE: Coronin 1B coordinates Arp2/3 complex and cofilin activities at the leading edge. Cell 128: 915-929, 2007.

29. Yan M, Di Ciano-Oliveira C, Grinstein S and Trimble WS: Coronin function is required for chemotaxis and phagocytosis in human neutrophils. J Immunol 178: 5769-5778, 2007.

30. Gandhi M, Achard V, Blanchoin L and Goode BL: Coronin switches roles in actin disassembly depending on the nucleotide state of actin. Mol Cell 34: 364-374, 2009.

31. Yokoyama K, Kaji H, He J, et al: Rab27a negatively regulates phagocytosis by prolongation of the actin-coating stage around phagosomes. J Biol Chem 286: 5375-5382, 2011.

32. Cai L, Makhov AM, Schafer DA and Bear JE: Coronin 1B antagonizes cortactin and remodels Arp2/3-containing actin branches in lamellipodia. Cell 134: 828-842, 2008.

33. Sawada N, Li Y and Liao JK: Novel aspects of the roles of Rac1 GTPase in the cardiovascular system. Curr Opin Pharmacol 10: 116-121, 2010.

34. Moissoglu K, Slepchenko BM, Meller N, Horwitz AF and Schwartz MA: In vivo dynamics of Rac-membrane interactions. Mol Biol Cell 17: 2770-2779, 2006.

35. Tomasevic N, Jia Z, Russell A, et al: Differential regulation of WASP and N-WASP by Cdc42, Rac1, Nck, and PI(4,5)P2. Biochemistry 46: 3494-3502, 2007. 